\section{La Révolution française}

Cahiers de l'Institut d'histoire de la Révolution française

$6 \mid 2014$

La Révolution ou l'invention de la femme et de I'homme nouveaux

\title{
Les républicains de Thermidor, ou produire du nouveau sans « homme nouveau »
}

\section{Ayşe Yuva}

\section{(2) OpenEdition}

\section{Journals}

Édition électronique

URL : http://journals.openedition.org/lrf/1124

DOI : $10.4000 /$ Irf. 1124

ISSN : 2105-2557

Éditeur

IHMC - Institut d'histoire moderne et contemporaine (UMR 8066)

Référence électronique

Ayşe Yuva, "Les républicains de Thermidor, ou produire du nouveau sans « homme nouveau » ", La Révolution française [En ligne], 6| 2014, mis en ligne le 28 septembre 2014, consulté le 14 février 2020. URL : http://journals.openedition.org//rf/1124; DOI : 10.4000/Irf.1124

Ce document a été généré automatiquement le 14 février 2020.

(c) La Révolution française 


\title{
Les républicains de Thermidor, ou produire du nouveau sans « homme nouveau »
}

\author{
Ayşe Yuva
}

1 «Régénérer » les hommes ou les «civiliser ", former un "homme nouveau »: ces expressions déjà courantes dans les dernières décennies de l'Ancien Régime ${ }^{1}$ deviennent récurrentes après 1789 , où l'on s'interroge sur la nature des changements produits en l'homme par la Révolution et les institutions nouvelles. Nous souhaiterions ici nous pencher sur la période thermidorienne, en analysant conceptuellement le type de nouveauté politique et anthropologique induite par la «rénovation» de l'entendement ou la «révolution dans les esprits» que certains des auteurs républicains de cette époque théorisent. Ceux-ci, certes, n'ont pas revendiqué le titre de philosophes, mais ont défendu la place de la philosophie dans les discours publics, les changements politiques et anthropologiques reposant en dernière instance sur les progrès des sciences, comme nous tâcherons de le montrer.

2 Mona Ozouf, dans L'homme régénéré, avait souligné que le clivage entre ceux qui évoquaient la possibilité d'une régénération instantanée et ceux qui privilégiaient le temps long ${ }^{2}$ ne suffisait pas à distinguer clairement les camps politiques en présence ${ }^{3}$; on pourrait ajouter qu'il ne nous révèle encore rien du type de nouveauté visé. Les républicains de Thermidor, prétendant «terminer la Révolution» en instruisant les esprits, en les mettant à la hauteur des lumières que la République suppose, renoncent certes à penser une régénération instantanée, une «inauguration absolue $»^{4}$ produite par la Révolution, mais non à former les hommes en vue d'un régime nouveau, la République. Mais cette nouveauté politique suppose-t-elle nécessairement, comme fin et principe, un homme lui-même nouveau ? Jusqu'à quel point le caractère inédit, dans un pays tel que la France, de ces institutions politiques, appelle-t-il un certain type d'homme et de citoyen dont l'histoire n'aurait pas montré l'exemple? Les républicains actifs durant le Directoire se distinguent non pas tant par leur projet pédagogique général, qui repose sur l'instruction sans s'y réduire, que par la façon dont ils articulent 
nouveauté morale, intellectuelle et scientifique : c'est le point que l'on suivra ici, en étant attentif aux temporalités mises ainsi en branle et à la façon dont les différents types de nouveauté s'inscrivent dans des processus historiques distincts quoique liés entre eux.

\section{Corruptions anciennes et présentes : la nouveauté des mœurs républicaines}

3 Chez ces auteurs dont le discours se caractérise par une prise de distance vis-à-vis de l'Ancien Régime aussi bien qu'une condamnation de la Terreur, l'homme et le citoyen que la République appelle sont pensés d'abord par opposition à d'autres figures qui, bien qu'antagonistes, ont pour point commun d'appartenir au passé et d'être bannies de l'avenir; toutefois le changement souhaité dans les mœurs n'induit pas nécessairement un projet de transformation de la nature humaine.

\section{Le dépassement de l'Ancien Régime}

Les auteurs du Directoire se situent dans la lignée républicaine par leur condamnation des mœurs et pour ainsi dire de l'esprit de l'Ancien Régime: ils reprennent le lieu commun selon lequel celui-ci est responsable d'une corruption qui pèse encore de tout son poids sur le présent. La responsabilité en incombe aussi bien aux institutions politiques qu'à l'Eglise, pouvoir spirituel associé au pouvoir de l'Etat ${ }^{5}$. Ce lieu commun selon lequel le chemin vers la régénération doit surmonter des obstacles hérités du passé $^{6}$ n'est caractéristique en lui-même ni d'une certaine période de la Révolution, ni d'un groupe d'acteurs. Cette critique ne doit cependant pas être comprise, par exemple chez Daunou, en un sens vaguement rousseauiste : les hommes ont été corrompus, non par la civilisation et par les lettres, mais malgré leur présence et du fait de leur instrumentalisation.

Il n'est cependant pas nécessaire, pour juger les hommes de l'Ancien Régime dépassés, d'aller jusqu'à les trouver moralement corrompus. Mme de Staël se contente de constater leur inadaptation aux nouvelles institutions; leur raffinement excessif ne correspond plus aux impératifs de l'époque présente. Elle rend justice à la grâce des manières, à l'esprit et au goût qui régnaient sous l'Ancien Régime et l'on a pu dire d'elle qu'elle reste attachée à une certaine culture aristocratique ${ }^{7}$; mais elle y voit, dans De la littérature, le corollaire d'un état de servitude politique; ainsi la délicatesse de l'honneur et la nécessité sociale d'avoir de l'esprit pour espérer s'élever socialement permettaient de "se courber sans s'avilir". » Ces mœurs étaient l'expression d'un certain compromis politique : le charme de la grâce et de la gaieté française de l'Ancien Régime sont le produit d'un gouvernement où tout dépend de la faveur d'un roi, qui luimême ne désire pas faire reposer son autorité sur la crainte mais veut lui donner des apparences de libéralité. La position de Germaine de Staël est intéressante en ce qu'elle reconnaît que cet esprit faisait, pour une bonne part, la supériorité de la France en Europe et expliquait que sa littérature et ses mœurs soient prises pour modèle par les autres nations. Mais outre que cette imitation mécanique de la culture française ne lui semble pas devoir perdurer, l'inactualité et la servitude politiques sont les deux raisons essentielles pour lesquelles cet esprit d'Ancien Régime est selon elle voué à disparaître. 
La «perfectibilité » est l'autre nom de cette inactualité des hommes et des régimes politiques du passé.

6 Celle-ci touche non seulement le courtisan, mais aussi, de façon peut-être plus paradoxale, l'écrivain s'adressant, contre le pouvoir, à l'opinion publique. Le caractère peut-être inattendu de cette critique réside dans le fait que Germaine de Staël comme Benjamin Constant prennent, dans le débat portant sur la responsabilité des philosophes dans le déclenchement de la Révolution, la défense des écrivains du XVIII ${ }^{\mathrm{e}}$ siècle qui ont cherché à se rendre « utiles » politiquement en faisant de leurs écrits des " armes »; mais cela n'empêche pas Constant de railler certains écrivains, survivances du passé, qui n'ont pas compris « qu'après sept années d'un bouleversement qui a usé toutes les forces, l'on ne pouvait avoir, pour leurs allusions fines, pour leurs nuances délicates, pour leurs piquantes épigrammes, l'empressement qu'on leur témoignait dans les temps paisibles et désœuvrés de la Monarchie ${ }^{9}$. ”

7 Dès le début de la Révolution - mais la tendance se poursuit sous le Directoire - la figure du «philosophe » voltairien «se ternit au profit du modèle plus énergique du législateur ${ }^{10} »$, ou, disons, de l'écrivain qui se définit avant tout comme républicain ${ }^{11}$. Les personnages de l'Ancien Régime, dont le "philosophe » et "l'homme de lettres » font partie, peuvent être jugés dépassés sans qu'il soit nécessaire pour cela de les percevoir comme corrompus. Les changements politiques survenus suffisent à les condamner, à les rendre inactuels.

\section{Révolution et dépravation des mœurs}

8 Pourtant, on aurait tort de penser que cette inactualité des personnages d'Ancien Régime revienne à adouber ceux produits, concrètement, par la Révolution, à une époque où nombre d'acteurs républicains se définissent en rejetant toute la responsabilité de la Terreur sur Robespierre et ses soutiens, devenus des "boucs émissaires » commodes ${ }^{12}$. Certes, les discours tenus par certains Thermidoriens sur les hommes du Comité de Salut public ou leurs soutiens sont sans doute parfois en contradiction avec leurs pratiques : il arrive qu'ils soient tenus par des "girouettes " qui taisent leurs compromissions passées ou occultent le maintien, juste après Thermidor, de certaines lois héritées de la Terreur ${ }^{13}$. Mais ces discours méritent néanmoins d'être lus pour eux-mêmes en ce qu'ils affirment précisément l'impossibilité de produire la régénération par l'épreuve, l'expérience de la Révolution. Si l'Ancien Régime interdisait aux individus d'être pleinement moraux, la Révolution ne l'a en effet pas permis davantage. Ces auteurs affirment avec force la coïncidence de la Révolution, non avec une quelconque régénération, mais au contraire une dépravation des mœurs; empiriquement, la Révolution, qu'il faut précisément «terminer », a produit selon eux une corruption accrue. Daunou, dont on a dit qu'il mettait en cause, en 1793, la formation des esprits sous l'Ancien Régime, déplore, dans son Rapport sur l'instruction publique de l'an IV, la « décadence» de l'instruction publique durant la Terreur ${ }^{14}$. Il contribue ainsi, encore une fois, à mettre à distance les principaux responsables de cette période, qui pourtant soulignaient eux aussi la menace de barbarie et présentaient justement la terreur comme un moyen de lutter contre la " dégénération ${ }^{15}$ $»$. 
9 Si la Révolution a produit la barbarie, est-ce alors du fait d'un mode de gouverner nouveau ou d'une dépravation déjà présente dans une certaine partie de la population, que ces auteurs élitistes désignent comme la " populace»?

Destutt de Tracy, dans son texte de 1798 intitulé Quels sont les moyens de fonder la morale chez un peuple?, nie que la Révolution soit en elle-même la cause de la corruption des mœurs ${ }^{16}$ : la concomitance des deux événements ne signifie pas qu'ils soient reliés par un rapport de cause à effet. Les institutions du temps présent n'ont pas le pouvoir de créer instantanément un homme nouveau - vertueux ou immoral. Pourtant, Destutt de Tracy refuse également de faire porter entièrement la faute de la dépravation sur l'Ancien Régime, puisque les mœurs et les idées auxquelles celui-ci a formé ont pu conduire à des comportements très différents, voire opposés avant et après 1789 . Destutt de Tracy souhaite expliquer le degré de dépravation, d'immoralité et de désordre - toutes choses liées entre elles - non par le régime politique, la nature monarchique ou républicaine des institutions, mais par le mode de gouvernement des hommes, la façon d'administrer le territoire : les désordres dans ce domaine conduisent à un moindre respect des "lois répressives", dans lesquelles Destutt de Tracy voit la meilleure garantie d'une moralisation des comportements. Le discours sur la dépravation ne relève pas, avant tout, d'un jugement portant sur la morale individuelle, mais est associé à une analyse de l'anarchie que l'on trouve également chez nombre de Thermidoriens ${ }^{17}$, dans un contexte qui suit d'ailleurs des années de répression pénale accrue ${ }^{18}$.

Ce faisant, Destutt de Tracy dédouane aussi bien le régime républicain que les théories philosophiques les ayant soutenues de toute responsabilité quant à la dépravation des mœurs. Cet état de fait ne signifie pas que la République a été mal pensée, mal théorisée. $\mathrm{Si}$ «la pratique de la morale s'est détériorée, quoique sa théorie se perfectionnait ${ }^{19}$ ", c'est parce que "l'application » de ces théories ne pouvait être faite instantanément, mais nécessitait une médiation législatrice et administrative qui n’a pu que difficilement être mise en place dans un temps de révolution, c'est-à-dire de changement de régime politique, qui a dû entraîner nécessairement, pour un temps, une désorganisation générale encourageant les comportements individuels. Mais parce que les lois répressives constituent un moyen certain de moralisation d'une société, le mal produit par la Révolution ne doit être que momentané. Par conséquent, la moralisation pourra également s'obtenir sans que l'on change la nature de l'homme.

12 En cela, Destutt de Tracy se distingue Germaine de Staël et Constant, qui voient au contraire dans «l'anarchie », "l'arbitraire » ou la dépravation des mœurs des maux inhérents à la Révolution (particulièrement à la Terreur) considérée comme moyen d'action politique, et que l'on ne pourra surmonter mécaniquement, ou par le seul respect extérieur des lois pénales. Certaines nouveautés produites par le changement politique brutal discréditent celui-ci comme instrument. Certes, on retrouve ici l'argument classique selon lequel la violence de la Révolution est due aux obstacles qu'elle a eus à renverser et non aux principes théoriques que l'on a tenté de mettre en place $^{20}$; mais rien ne dit que les maux produits soient tous provisoires et que l'état d'esprit révolutionnaire ne constitue pas encore une menace pour le présent. L'héritage de la Révolution se voit en effet dans l'apparition d'hommes « nouveaux » qui n'ont pas disparu avec la fin de la Terreur.

13 Les hommes que l'on a vu concrètement agir sous la Révolution, sous la figure individuelle $\mathrm{du}$ démagogue et $\mathrm{du}$ révolutionnaire, ou celle, collective, de la 
"populace ", ont été, selon ces auteurs, porteurs d'une corruption inédite, caractérisée par des manières qui pour être "nouvelles" n'en doivent pas moins être rejetées. Constant, dans De la force du gouvernement actuel et de la nécessité de s'y rallier ${ }^{21}$, distingue les hommes formés au persiflage de l'Ancien Régime des « hommes nouveaux, violents, énergiques, qui avaient appris à braver plus que le danger, et dont le caractère avait été formé par la plus terrible éducation révolutionnaire ${ }^{22}$." La Révolution a été en ellemême une éducation morale, forgeant, dans l'action, des hommes dont l'Ancien Régime n'avait pas montré l'exemple. Les événements ont révélé des facettes jusque-là cachées du caractère humain. Les terroristes, produits de cette éducation révolutionnaire, sont, encore après la Terreur, l'arme secrète des Républicains qui garantit la pérennité du nouveau régime :

Ces hommes, ou plutôt ces êtres, d'une espèce inconnue jusqu'à nos jours, phénomènes créés par la Révolution, à la fois mobiles et féroces, irritables et endurcis, impitoyables et passionés [sic], qui réunissent ce qui jusqu'à présent paraissait contradictoire, le courage et la cruauté, l'amour de la liberté et la soif du despotisme, la fierté qui relève et le crime qui dégrade, ces tigres (...), cette race nouvelle, qui semble sortie des abîmes pour délivrer et dévaster la terre (...) ces puissances aveugles de destruction et de mort, ont mis au retour de la Royauté un obstacle qu'elle ne surmontera jamais ${ }^{23}$.

14 La nouveauté est ici monstruosité, contradiction logique incarnée dans un régime, la Terreur, qui avait fait de l'oxymore qu'est le «despotisme de la liberté » sa devise, et qui a produit des hommes qui lui ressemblent, réunissant des traits de caractère que l'on croyait incompatibles entre eux. Le joug politique pourrait être surmonté s'il n'était en même temps accompagné de cette monstruosité morale qui compromet l'avenir des institutions politiques. Ici, Constant reprend en quelque sorte le modèle platonicien classique selon lequel le tyran, parce qu'il ne sait gouverner son âme, ne gouverne les hommes que de façon elle-même tyrannique: dans le cas de la Terreur, l'arbitraire et la violence ont eu à la fois comme principe agissant et comme résultat des hommes moralement aussi contradictoires que ce mode de gouvernement. Alors que dans De la force du gouvernement actuel, Constant envisage encore dans quelle mesure ces hommes d'un nouveau genre pourraient servir d'appui aux républicains, dans Des effets de la Terreur, où "l'arbitraire " est pris pour cible, il soutient que les révolutionnaires de la période précédente, loin d'affermir la République, ont contribué à son discrédit; la Terreur "a courbé les têtes, mais elle a dégradé et flétri les cœurs ${ }^{24}$. " L'obéissance extérieure est superficielle et les effets moraux des modes de gouverner se révèlent plus durables et potentiellement plus dangereux qu'une contrainte exercée sur les comportements.

15 Les classes populaires, jugées ignorantes car non instruites ni cultivées, peuvent encore moins prétendre incarner une nouveauté désirable ${ }^{25}$. Daunou conspue ceux qui flattent le peuple en lui faisant croire qu'il est déjà « libre et régénéré ${ }^{26}$. Mme de Staël invente le néologisme de "vulgarité » pour dire les effets de l'irruption des classes populaires sur le devant de la scène politique - qui se sont fait ressentir sur les mœurs, les manières, le style des discours et des écrits. La présence d'un acteur collectif, le peuple, et des individus qui le composent est jugée illégitime dans l'espace public. Cette condamnation est justifiée au nom des lumières et du degré d'instruction : le règne de la vulgarité équivaut à la domination politique, morale, symbolique des hommes non éclairés. Ceci est jugé contraire aux principes de la République, régime dont les institutions sont rationnelles et où les citoyens ne doivent plus être soumis purement et simplement à la contrainte, mais savoir se conduire eux-mêmes, et dont les membres 
actifs, c'est-à-dire de facto les élites dirigeantes, doivent être instruites. C'est pourquoi la question de la formation intellectuelle, des lettres et du goût est directement politique : elle doit servir à distinguer les acteurs qui peuvent légitimement occuper le devant de la scène politique et prendre la parole publiquement des autres. En remettant les hommes éclairés au pouvoir, Mme de Staël entend, du même mouvement, mettre au premier plan des manières et des sentiments qui soient dignes du nouveau régime républicain.

Cependant, si la Révolution n'est pas un moyen de régénération, la réaction, son pendant monarchiste, qui ne serait d'ailleurs rien d'autre qu'une nouvelle révolution ${ }^{27}$, ne l'est pas davantage: les auteurs ici envisagés ne se conçoivent pas comme des réactionnaires, mais des républicains capables d'asseoir enfin le nouveau régime politique sur des bases solides, après la séquence de la Terreur qui a signifié un recul de la moralité et des libertés individuelles. Cette prise de distance avec la Révolution est aussi le rejet d'un retournement par trop radical des manières, des mœurs et des discours, qui ne correspond pas à l'essence profonde de la République. Les institutions politiques ne peuvent ni ne doivent produire seules le changement désiré et ont besoin d'un adjuvant qui consistera dans une certaine direction donnée aux esprits.

\section{La formation des esprits par-delà l'instruction publique}

17 Si la Révolution s'est accompagnée d'une certaine dépravation des mœurs (ou si du moins elle a été incapable d'y remédier), cela ne signifie pas, en effet, que l'on doive renoncer à toute perspective de changement, non pas de l'homme, mais en l'homme. Les institutions de l'Ancien Régime allaient de pair avec un raffinement proche de la corruption ; l'anarchie et la démagogie révolutionnaires ont encouragé la dépravation ; la stabilité des institutions républicaines doit être le point de départ d'une nouvelle formation - car il ne s'agit pas tant de former un homme entièrement nouveau que de faire disparaître certains types d'hommes asservis car ignorants, de changer les hommes en les instruisant à l'échelle de la société. Les auteurs ne renoncent pas à l'idée de former un "esprit public" républicain, inédit en ce qu'il ne se ramène pas simplement à un modèle antique. Les auteurs du Directoire partagent avec les Républicains de 1789 la conviction que les esprits doivent être à la hauteur des institutions, que ces deux éléments se conditionnent mutuellement. Mais de fait, pour eux, de nombreux désordres révolutionnaires survenus - principalement à partir de 1792 - s'expliquent par le décalage existant entre les deux : « La République s'est établie en France cinquante ans avant que les esprits y fussent préparés : on a eu recours à la Terreur pour l'établir ${ }^{28}$.» Le paradoxe est que la Révolution, bien qu'ayant été provoquée par les écrits des philosophes - et ayant de ce fait un caractère " philosophique »- est survenue avant que ceux-ci aient pu être réellement assimilés par la population. L'enjeu est de rétablir le rapport de convenance requis entre l'état des lumières et celui des institutions républicaines : ainsi que le résume Pierre Serna, "la tâche de ces écrivains est concrètement d'inventer une culture républicaine capable de faire changer efficacement des mentalités restées encore largement imprégnées de l'esprit d'avant 1789 , avec ses marques de soumission servile à la royauté ou de contestation brutale de l'ordre ${ }^{29}$. » Les élites éclairées s'attribuent ici une « mission civilisatrice ${ }^{30}$ » si l'on veut, mais non pas de régénération. 


\section{L'insuffisance d'une formation intellectuelle et intérieure des individus}

De fait, la formation envisagée sera essentiellement intellectuelle. Nombre d'acteurs de cette époque ont été, comme on le sait, des penseurs majeurs de l'instruction publique - dont des institutions comme les écoles centrales ou l'école normale de l'an III sont devenues les emblèmes. Héritiers de Condorcet sur ce point, les Thermidoriens se méfient d'une formation du «cœur» qui se ferait au détriment de celle de l'entendement. Cela ne signifie pas que la portée des lumières soit uniquement théorique ou ne concerne que les sciences : les auteurs tentent précisément de penser comment une formation intellectuelle peut donner lieu à une " habitude de la raison ${ }^{31}$ " nouvelle, c'est-à-dire avoir une portée pratique effective.

Mais à rebours de l'image convenue de ceux que l'on a appelés plus tard les "Idéologues", présentés comme des tenants de l'instruction publique, voire des précurseurs de la $\mathrm{III}^{\mathrm{e}}$ République, nous présenterons ici plusieurs cas où celle-ci est mise à l'arrière-plan et où les autorités politiques s'abstiennent d'agir directement sur les esprits individuels.

Destutt de Tracy distingue ainsi les effets de l'instruction publique, toujours lointains, et les effets plus immédiats de la législation pénale et des actes d'administration, qui ne changent certes pas les hommes mais influent directement sur leur comportement extérieur :

Les législateurs et les gouvernants, voilà les vrais précepteurs du genre humain, les seuls dont les leçons aient de l'efficacité ; l'instruction morale surtout, on ne saurait trop le répéter, est tout entière dans les actes de législation et d'administration ${ }^{32}$.

21 Certes, les écoles ne sont pas rejetées comme vecteurs de changement; la fin visée demeure de faire prendre conscience aux individus de la convergence de leurs intérêts et de permettre ainsi l'émergence de rapports plus conformes à la «nature ». Mais l'instruction publique et la diffusion des lumières nécessitent de se projeter sur un temps long, là où la promulgation des lois ne demande qu'un législateur bien conseillé par les savants et les philosophes. L'instruction court toujours le risque d'être trop théorique, là où les lois ont des effets directs sur les actions, et même les mœurs et les habitudes. Par exemple, en autorisant le divorce, on contribuera au bonheur des ménages plus sûrement que par des sermons : «les lois sont l'éducation des hommes faits $^{33}$ ", redira encore Destutt de Tracy dans un texte plus tardif.

Un décalage d'un autre type se voit dans l'Essai sur l'instruction publique de Daunou de 1793, où son insistance sur l'importance des fêtes ne signifie pas une dépréciation de la formation intellectuelle et ne contredit pas ses textes ultérieurs datant du Directoire. En effet, c'est au nom de la liberté qui doit être laissée aux individus dans ce domaine que Daunou y limite l'intervention de l'Etat. Une instruction des esprits pourrait trop facilement se transformer en inculcation de nouveaux dogmes. Les fêtes, de ce point de vue, lui semblent moins dangereuses; elles sont même propices à la "régénération " qui signifie ici une liberté garantie par les institutions ${ }^{34}$, un état caractérisant donc un régime politique plutôt que les esprits individuels. Daunou est, au contraire, assez proche des critiques formulées par G. de Staël et B. Constant à l'encontre d'une emprise de l'Etat sur les opinions individuelles et la formation des esprits. Le gouvernement n'a pas à régénérer les hommes en les formant intellectuellement ou moralement ${ }^{35}$, mais à 
offrir les conditions politiques par lesquelles les individus pourront se former selon des processus sociaux, interindividuels indépendants de l'Etat.

\section{La rhétorique philosophique et la « nouvelle » littérature républicaine}

Staël et Constant, cependant, préconisent à l'époque du Directoire une voie originale en se détournant aussi bien de l'instruction publique que des fêtes: les écrivains «font marcher l'esprit public plus vite et plus loin qu'une éducation nationale ${ }^{36}$.» Bien que collaborant avec le gouvernement, c'est-à-dire œuvrant pour une paix fondée sur les principes républicains, ceux-ci doivent être institutionnellement indépendants. Ils diffusent "l'idéologie» de la République - au sens vague de ce mot - mais sans participer directement au pouvoir de l'Etat ni même être rattachés à une institution. Dans la perspective Germaine de Staël et Constant, cette collaboration se fait en toute liberté et dans une visée qui n'est pas de domination, mais tout de même de direction des esprits: les écrivains qui n'écrivent qu'au nom de leurs convictions et de leur conscience morale, animés par le désir de se rendre utiles à la collectivité, pourront contribuer à former «l'opinion publique » dans le sens désiré et propager les lumières sans que la liberté des individus soit niée. Le dirigisme intellectuel qui use de l'art des discours n'est pas perçu comme une contrainte, dans la mesure où l'éloquence n'est persuasive que pour autant qu'elle s'appuie sur des vérités démontrées ou des évidences morales. La voie choisie par Mme de Staël et B. Constant est celle des « discours ", d'une certaine rhétorique philosophique sommée de pacifier la société en réunissant les individus autour de vérités scientifiques. Cependant il importe de savoir présenter adéquatement ces calculs et ces démonstrations qui gagnent ainsi en force ; les discours ne sont pas un moyen extérieur de présentation, mais déjà une manifestation sensible par laquelle on peut remonter aux vérités : " les mots ont sur nous une telle influence qu'ils ramènent les idées", car "les formes perpétuent l'esprit ${ }^{37}$. » L'usage démagogique des mots est loin d'être le seul possible. On peut même dire que faute de mots et de formes, le changement politique est incapable d'amener la liberté politique, ainsi que l'exemple de l'Asie, qui a connu de nombreuses "révolutions », en témoigne selon eux. Il est donc requis pour la formation des citoyens que les écrivains et les gouvernants mettent des mots sur les libertés nouvelles.

C'est pourquoi il est nécessaire d'avoir, sous un régime politique nouveau, une littérature également nouvelle. La seconde partie de De la littérature ${ }^{38}$ est précisément une esquisse des traits de la société et de la littérature républicaines à venir. Parce que les lettres et les institutions se soutiennent mutuellement, parce ces phénomènes sont englobés dans le processus général de perfectibilité, on retrouve des schèmes similaires pour penser le changement dans les deux domaines: Mme de Staël nie ainsi qu'une "révolution » soit nécessaire dans le domaine de la littérature ${ }^{39}$. Le régime républicain appelle des magistrats dont le langage et les manières soient empreints de « dignité » et qui puissent servir de modèles aux autres citoyens. Parce qu'il existe des affinités entre les deux républiques nouvellement constituées, elle cite en exemple un éloge funèbre de Washington - tout en soulignant les écarts entre ce discours et les harangues révolutionnaires. Cette insistance sur la dignité et la simplicité montre les difficultés que Mme de Staël éprouve, malgré tout, à se détacher du modèle des républiques antiques aux mœurs sévères et frugales. 

pas par rupture avec le premier et il est nécessaire de suivre une voie médiane, entre rupture révolutionnaire et répétition de ce qui existe déjà, en littérature comme en politique. Ces sphères, loin d'être autonomes, établissent entre elles, au sein de chaque société, un rapport de "convenance " et se soutiennent mutuellement dans leurs progrès respectifs, qui s'insèrent dans l'histoire plus générale de la perfectibilité de l'esprit humain. Parce que la voie politique juste se trouve entre l'Ancien Régime et la Révolution, Mme de Staël préconise en littérature d'éviter aussi bien la création ex nihilo, les «mots nouveaux », « l'énergie du style » que la répétition du même, le « style exact, mais commun ». Dans la querelle des Anciens et des Modernes, Mme de Staël penche clairement en faveur des seconds (sauf pour le domaine de la poésie); d'ailleurs les modernes d'un passé récent doivent eux aussi être dépassés: la philosophie républicaine dont la France a besoin ne s'est incarnée ni au XVIII ${ }^{e}$ siècle, ni dans la Rome antique. Certes, Mme de Staël accorde une valeur indéniable à «l'originalité » et la «création» de l'écrivain. Mais la définition de cette nouveauté doit être précisée : l'écrivain génial n'est pas celui qui rompt avec tout ce qui a été créé avant lui; car le génie, c'est-à-dire celui qui produit des œuvres véritablement nouvelles, ne le fait qu'à partir d'une connaissance du passé, et même d'une reprise de certains de ses éléments. En ce sens, Mme de Staël ne s'interdit pas de trouver des traits dignes d'être repris chez certains écrivains du XVII et du XVIII ${ }^{e}$ siècle : Montesquieu, Rousseau et Condillac sont considérés comme des républicains avant l'heure ${ }^{40}$, Racine est donné en exemple pour ce qui est de la pureté du style et les œuvres de certains écrivains, tels Molière, semblent avoir, par la connaissance de l'homme dont elles témoignent, une valeur universelle.

Plus tard, dans De l'Allemagne, Mme de Staël montrera que les racines de la corruption morale sont plus anciennes que la Terreur même et se trouvent dans un certain «matérialisme » du XVIII siècle (qu'elle définit par la conjonction du matérialisme ontologique, de l'empirisme de la connaissance et de l'utilitarisme moral), auquel elle tentera d'opposer la pureté de l'idéalisme allemand; elle prendra alors ses distances avec les Idéologues, ne considérant plus leur philosophie comme le remède dont l'époque a besoin ou le germe de la philosophie de l'avenir, mais au contraire la continuation de cette immoralité ancienne. Elle se tournera vers les pays du Nord plutôt que vers le passé proche afin, toujours, de renouveler la littérature, et partant les esprits, en s'appuyant toujours sur une certaine conception de l'homme: et en critiquant le «fondement anthropologique de la politique napoléonienne, à savoir un parti pris "machiavélien" qui suppose qu'on puisse toujours parier sur l'égoïsme humain et la manipulation des êtres en fonction de leur intérêt personnel ${ }^{41}$. " L'idée demeurera donc que l'éveil de la moralité humaine et le changement politique via l'action de certains individus mus par l'enthousiasme sont rendus possibles par la diffusion de certains discours philosophiques. Mais ce qui inspirera cette nouveauté sera l'ouverture à l'étranger.

\section{La « rénovation » scientifique et l'homme « nouveau »}

L'idée même de perfectibilité consiste certes, chez Condorcet, mais aussi plus tard chez de Staël et Constant, à «tracer des perspectives sans tomber dans un imaginaire qui présupposerait une altérité radicale dans l'organisation de la société comme dans la 
nature de l'homme ${ }^{42}$.» A l'époque du Directoire, le projet politique de changement des esprits ne repose pas sur l'idée que l'Etat aurait à produire un "homme nouveau »: l'émergence d'une société nouvelle ne nécessite pas un détour aussi coûteux ${ }^{43}$. Et les vecteurs mobilisés, pour être de nature politique, ne relèvent pas nécessairement directement de l'Etat.

De même que les institutions politiques ne produisaient pas d'elles-mêmes le citoyen dont elles avaient besoin, de même le mécanisme d'un gouvernement des esprits " par le haut » n'est pas assuré de son efficace s'il ne repose sur des bases théoriques solides. Celles-ci consisteront avant tout, sous le Directoire, dans des projets de connaissance de l'homme via les «sciences morales et politiques », «l'idéologie » puis l'anthropologie, ainsi que J.-L. Chappey l'a analysé. Le politique peut et doit s'appuyer, dans sa volonté de changement, sur une certaine connaissance de la nature humaine, elle-même prise dans l'histoire des sciences. Or certains acteurs n'avaient pas manqué de souligner dès 1789 l'urgence de progrès scientifiques dans la connaissance de l'homme à des fins de gouvernement ${ }^{44}$, soulignant le décalage entre ces derniers et l'histoire politique. Qu'en est-il chez les penseurs du Directoire?

\section{Rénovation philosophique et révolution politique}

Qu'il agisse au moyen des discours, de l'instruction publique ou des lois, le membre du gouvernement doit disposer d'une certaine connaissance de la nature humaine empirique - par l'idéologie ou "science des idées" mais également au moyen de "l'anthropologie ${ }^{45}$ ». Dans le cas des lois pénales, en particulier, il ne s'agit pas tant de créer des hommes ou des esprits nouveaux que de s'appuyer sur certains mécanismes régulant les comportements. Destutt de Tracy n'espère pas changer les intentions des individus mais accorder extérieurement les différentes volontés entre elles, de sorte qu'elles respectent les normes morales: par exemple, il estime démontrer que les individus ont des intérêts distincts qui peuvent éventuellement s'opposer; on ne peut détruire l'idée de toi et de moi, de tien et de mien - dont on peut au contraire tirer l'idée de propriété et certaines mesures économiques ou juridiques. Le philosophe met cette connaissance "naturelle » de l'homme à la disposition du législateur - Destutt de Tracy composera un Traité de la volonté et de ses effets, publié en 1818, dans lequel il proposera encore une théorie de l'homme à l'usage du gouvernement - la théorie de l'économie politique faisant alors, ce qui n'était pas encore le cas en 1798, la jonction entre la théorie de l'homme et la science politique :

J'aurais laissé aux philosophes et aux législateurs à tirer les conséquences de ces données et à proposer les lois politiques, civiles, morales et pénales les plus propres à développer nos talents et nos vertus, à étouffer ou à comprimer nos mauvais penchants et à assurer notre bonheur ${ }^{46}$.

La science de l'homme doit, en 1798, guider directement les mesures des dirigeants en vue d'un gouvernement des passions. Destutt de Tracy remarque que si les lois font les citoyens, il est néanmoins nécessaire pour cela que les législateurs possèdent «la théorie méthodique de la morale domestique et sociale ${ }^{47}$. " Ainsi, les progrès de la philosophie et particulièrement des sciences qui concernent l'homme s'avèrent être une condition décisive pour la formation nouvelle des hommes et des citoyens. changement immédiat et instantané est déprécié tout comme en politique ${ }^{48}$. Destutt 
de Tracy refuse de penser l'histoire des sciences en termes de changement immédiat. En philosophie ou dans les sciences, une nouveauté radicale ne saurait être l'œuvre d'un instant (ou d'un unique individu). Ni Bacon, ni Descartes, ni Locke n'ont accompli cette « rénovation » ou ce « renouvellement» de la philosophie, qu'ils se sont contentés d'initier :

[Lorsque Bacon] sentit et proclama qu'il fallait refaire l'esprit humain, lui-même, avec tout son génie, ne put y réussir; il n'entrevit que confusément le moyen d'opérer cette refonte. L'esprit humain n'était point connu, et une science ne se fait pas tout d'un coup, surtout celle-là ${ }^{49}$.

Par contre, une fois un changement initié, celui-ci peut être approfondi dans la durée. Ainsi, la science "neuve" de l'idéologie s'inscrira bien dans une certaine lignée philosophique. L. Clauzade, dans son anthologie des Idéologues, note même que les cours donnés par Garat à l'Ecole normale supérieure exposent «un condillacisme fidèle » qui « donne le sentiment étrange qu'il cherche à donner un nom nouveau à une science déjà advenue ${ }^{50}$. $\gg$ Destutt de Tracy est sans doute davantage soucieux de critiquer ces œuvres; mais ce sera, d'une certaine façon, pour mieux les continuer. Même Cabanis, qui, en insistant sur l'influence des organes, semble rompre d'un point de vue épistémologique avec Condillac, s'autorise de certains précurseurs, philosophes médecins, tels que Locke ${ }^{51}$.

On peut certes citer des cas où non seulement le schème de la Révolution semble réévalué, mais où les événements politiques et philosophiques sont étroitement articulés : ainsi, Lakanal, dans son Rapport sur l'établissement des écoles normales, met-il en parallèle révolution politique et spirituelle en affirmant que l'instruction doit «terminer la révolution dans la République française, et en commencer une dans l'esprit humain ${ }^{52}$.» Ce parallèle s'accompagne d'ailleurs, ce qui n'est pas un hasard, d'une référence à la « régénération de l'entendement humain ${ }^{53}$ » - et non de l'homme, notons-le par parenthèse - à la " restauration ", la « recréation » de l'esprit humain ; ce qui se fera par la « diffusion institutionnelle et politiquement réglée du savoir ${ }^{54}$. » Mais rappelons ici l'ambiguïté du terme de "révolution», qui désigne certes, chez ces auteurs, un changement profond des structures, mais n'indique pas nécessairement que celui-ci soit opéré rapidement ou par le seul changement institutionnel. Lakanal n'exclut d'ailleurs pas le schéma d'une accumulation progressive des connaissances qui prélude à leur diffusion. La "révolution " peut s'inscrire dans le temps long de la perfectibilité : ainsi Mme de Staël dira qu'il faut achever la « révolution » initiée dans les lettres par Rousseau, Montesquieu et Condillac ${ }^{55}$.

La République dispose-t-elle cependant d'ores et déjà de la théorie et de la science susceptibles d'éclairer les mesures en vue de diriger et former les hommes? La condition à la mise en place de l'instruction publique est que «l'esprit humain ait fait assez de progrès pour être sûr de posséder les méthodes et les instruments avec lesquels il est facile d'éclairer tous les esprits et de faire tous les progrès ${ }^{56}$. » Là encore, Lakanal se distingue par son optimisme quant aux effets de la Révolution : les écoles normales doivent précisément former les futurs instituteurs à ces "méthodes». L'instruction publique apparaît comme le lieu d'une récapitulation :

«C'est le moment où il faut rassembler dans un plan d'instruction publique [...] les lumières accumulées par les siècles qui nous ont précédés, et les germes des lumières que doivent acquérir les siècles qui nous suivront ${ }^{57}$. « idéologie », pourtant partie prenante du projet républicain ${ }^{58}$. Mais la portée politique 
de cette science ne doit pas faire croire à l'existence d'un processus historique unique : si c'est à partir de cette "science des idées" que l'on pourra dériver l'art de la législation et de l'instruction ${ }^{59}$, l'apparition de la première n'est pas corrélée à la révolution politique française. Elle plonge ses racines dans l'œuvre d'auteurs du passé Bacon, Descartes, Locke et Condillac, pour ne citer que les noms les plus importants. L'entreprise scientifique passe régulièrement par une "refonte » de l'esprit humain, que l'on pourrait en quelque sorte comparer à un changement de paradigme - mais en se déliant, encore une fois, du processus de changement politique. On a ici affaire à des histoires qui ne sont pas sans rapport, mais ne se conditionnent pas mutuellement et peuvent se penser de façon autonome.

Une tension existe donc entre cette histoire des sciences et l'efficace politique qui leur est attribuée : le caractère inachevé de ce savoir rejaillit sur le régime républicain qu'il est censé soutenir. L'analyse scientifique de Tracy se distingue du discours - sans doute de nature plus directement politique - d'un Lakanal qui considère comme acquis certains résultats (dont on pourra seulement trouver des démonstrations plus " évidentes ») sur lesquels le régime républicain fonde sa légitimité. Ce dernier se place, encore une fois, dans l'horizon d'un changement immédiat et d'une «recréation » de l'entendement :

Bacon, Locke, en approfondissant [la nature de l'esprit humain], ont trouvé tous ses moyens de direction ; un nouveau jour s'est répandu sur les sciences qui ont adopté cette méthode si sage et si féconde en miracles ${ }^{60}$.

Ce dernier terme de «miracle» serait, sans nul doute, banni par Destutt de Tracy; l'idée même d'une trouvaille complète et définitive lui est à vrai dire étrangère. Non seulement la révolution scientifique n'est pas " parallèle » à la révolution politique en ce qu'elle reprendrait les modalités de changement de cette dernière, mais les progrès scientifiques qui doivent servir à l'affermissement du régime né de la Révolution sont loin d'être acquis. La République comme la science des idées ou de l'homme sont encore en construction, et même à l'état de projet : non seulement la révolution politique ne se transpose pas dans le champ de la science, mais l'inachevé de cette dernière se retrouve dans le champ de la législation et du gouvernement. Ce caractère de construction progressive touche même la morale qui, loin d'être douée d'un caractère immédiat, vient, en raison de son caractère complexe et composé, en dernier dans l'ordre des sciences, et doit être achevée en dernier ${ }^{61}$. Ce phénomène qui était, chez Condorcet, considéré comme la manifestation du caractère perfectible de la République peut aussi être perçu comme l'indice d'une fragilité - et nourrir, sous le régime instable du Directoire, des inquiétudes légitimes.

\section{La connaissance de la nature humaine et les principes moraux}

Dans le projet «idéologique », la connaissance des idées et plus généralement de la nature humaine devrait, idéalement, guider le législateur, le pédagogue et faire l'objet, au moins en partie, d'un enseignement touchant aux questions de morale dans l'instruction publique. Mme de Staël et Constant, à l'époque du Directoire, ne doutent pas de la légitimité d'un tel projet, malgré leur méfiance envers l'instruction publique. Mais ils hésitent à cette époque sur la possibilité de fonder la moralisation de la société sur une telle connaissance théorique de ce que l'on appellera un temps «les sciences morales et politiques» ou la "science politique». Staël, dans Du gouvernement des passions, en 1796, essaie de penser comment un tel gouvernement est possible, à 
l'échelle collective, par le biais de sciences telles que la statistique ou la science politique - tandis que la philosophie n'agit que sur certains esprits individuels, dans une perspective classique de gouvernement et de maîtrise de soi. En 1798, dans l'ouvrage non publié Des circonstances actuelles, il semble que la conduite intérieure des individus soit obtenue de façon sinon plus efficace, du moins plus rapide, par le biais d'idées religieuses et de principes moraux - les deux étant indissolublement liés. Il ne s'agit pas tant de concilier les intérêts individuels que de montrer la possibilité de dépasser toute pensée de l'intérêt par une prise de conscience de principes moraux suprasensibles et inconditionnés. La science de l'homme a pour pierre de touche les vérités de la morale, qui elles-mêmes peuvent être renforcées par des démonstrations, des évidences logiques ou des calculs. La conscience morale a alors cet avantage sur la science politique démonstrative qu'elle n'est précisément pas à l'état de projet. Staël et Constant choisissent de s'appuyer sur ces facteurs spirituels et liés au sentiment; si régénération il y a, elle se fera uniquement sous la forme d'un rappel, d'une actualisation de principes moraux inscrits dans le cœur de tout homme.

La morale, liée à la religion, non pas dans son versant dogmatique mais dans celui du sentiment, ne se résume pas à un ensemble de propositions et de vérités closes, car certains faits comme le caractère injuste de l'esclavage ne sont découverts que progressivement dans l'histoire. Mais cette perfectibilité dans la découverte de vérités politiques et morales a pour fondement un fait anthropologique intemporel sur lequel Staël et Constant mettront particulièrement l'accent dans la seconde partie de leur œuvre : la possibilité du sacrifice du présent. Il ne s'agit pas tant de penser un homme ni même une morale nouveaux que de faire prendre conscience à l'individu de son perfectionnement possible, qui repose sur la possibilité d'un sacrifice des intérêts présents. A l'époque du Directoire, ces auteurs croient encore à la possibilité de traduire ces principes moraux en une science susceptible de guider la politique du gouvernement, sans abolir la capacité d'action des gouvernés. L'individu doit pouvoir avoir recours à cette force intérieure pour agir ; il n'a pas besoin d'être formé par l'Etat, mais surtout d'être soutenu par sa conscience. C'est pourquoi la charge des magistrats et des écrivains sera de mettre en place une rhétorique qui éveille la conscience morale des lecteurs et des auditeurs. Le «naturel » que Mme de Staël juge digne des manières et des discours républicains est précisément une expression sans artifice, mais non sans éloquence, des idéaux de la morale. Ceci est une idée que Mme de Staël continuera à défendre, notamment dans De l'Allemagne, où elle mettra en avant des discours qui, sans cesser d'être philosophiques et rationnels, peuvent être éloquents, s'unir au sentiment religieux, et finalement contribuer à un réveil spirituel et moral - auquel elle appelle dans la conclusion de l'ouvrage, censurée par le pouvoir impérial.

Est donc déniée à la Révolution la capacité à incarner et produire par elle-même la nouveauté en l'homme: elle n'a donné naissance qu'à des individus affichant une nouvelle forme de corruption et a donné jour à un régime certes inédit mais dont la stabilisation requiert des moyens autres qu'institutionnels. L'établissement d'institutions politiques nouvelles ne suffit pas: la formation des esprits et leur moralisation ne doivent pas être confondues avec une "régénération » instantanée, et ce d'autant plus que ces penseurs inscrivent, en politique comme en science, la nouveauté même radicale dans le temps long de progrès cumulés. Les républicains du Directoire, lorsqu'ils ne veulent pas se contenter de réguler les comportements par les lois, pensent tout de même comment user de la nature humaine à des fins politiques. 
Mais si celle-ci a besoin d'être connue du législateur, la fragilité dans l'avancée des sciences de l'homme peut sembler inquiétante ${ }^{62}$. C'est la raison pour laquelle l'éveil toujours possible d'une conscience morale liée à des principes suprasensibles apparaît également, dès cette époque, comme une solution envisageable pour assurer le gouvernement des hommes.

\section{NOTES}

1. Voir Jean-Luc CHAPPEY, «De la science de l'homme aux sciences humaines: enjeux politiques d'une configuration du savoir (1770-1808) », Revue d'histoire des sciences humaines, 2006/2, $\mathrm{n}^{\circ} 15$, p. 43-68.

2. Mona OZOUF, L'homme régénéré, Paris, Gallimard, 1989, p. 119.

3. Ibid., p. 146.

4. Florence LOTTERIE, Progrès et perfectibilité : un dilemme des Lumières françaises (1755-1814), Oxford, Voltaire Foundation, 2006, p. XXVIII, où elle évoque la "mythologie de l'instantanéité » des Montagnards: «La Révolution est inauguration absolue, et toute pédagogie doit se faire accompagnement d'une révélation propre à l'ensemble de la cité. »

5. Voir Pierre-Claude-François DAUNOU "Rapport sur l'instruction publique », dans Bronislaw ВасZко (dir.), Une éducation pour la démocratie. Textes et projets de l'époque révolutionnaire, Genève, Droz, 2000, p. 506 : «Le même gouvernement qui courboit les peuples sous l'ignominieux empire de toutes les erreurs, avilisoit davantage encore les hommes les plus éclairés, en les plaçant avec lui dans la sphère de toutes les immoralités et de tous les vices. »

6. Voir Pierre-Claude-François DAUNOU, «Essai sur l'instruction publique », dans Bronislaw BACZKO (dir.), op. cit., p. 308 : «Lorsqu'il s'agit en effet d'un peuple immense qui, longtemps corrompu par le despotisme, conduit à la réforme de son organisation par l'excès de la dépravation commune, il n'arrive à la liberté qu'avec l'habitude et la tradition de tous les vices, et ne consomme sa régénération politique qu'au milieu des orages et des secousses de l'anarchie : certes, on voit trop qu'un tel peuple a plus besoin encore de mœurs que de lois, et d'instruction que de gouvernement. »

7. Voir Bronislaw BACzKo, Politiques de la Révolution française, Paris, Gallimard, 2008, p. 389 sq.

8. Germaine de STAËL, De la littérature, Paris, GF, 1991 [1800], p. 273.

9. Benjamin CONSTANT, "Des réactions politiques", dans De la force du gouvernement actuel de la France et de la nécessité de s'y rallier. Des réactions politiques. Des effets de la Terreur, Paris, Flammarion, 1988, p. 112 (Seul le titre du texte dont est tiré l'extrait sera dorénavant cité).

10. Jean-Claude BONNET, introduction de partie dans Jean-Claude BONNET (dir.), La Carmagnole des Muses. L'homme de lettres et l'artiste dans la Révolution, Paris, Armand Colin, 1988, p. 297.

11. Jean-Claude BONNET, « la sainte masure, sanctuaire de la parole fondatrice », dans J.-C. BONNET (dir.), op. cit, p. 212.

12. Voir Pierre SERNA, La république des girouettes, Seyssel, Champ Vallon, 2005, p. 365 et p. 377.

13. Ibid.

14. Pierre-Claude-François DAUNOU, « Rapport sur l'instruction publique », dans Bronislaw BACZKO (dir.), op. cit,, p. 507. 
15. Voir Jean-Luc CHAPPEY, « De la science de l'homme aux sciences humaines: enjeux politiques d'une configuration du savoir (1770-1808) », Revue d'histoire des sciences humaines, 2006/2, $\mathrm{n}^{\circ} 15$, p. 53-54.

16. Antoine-Louis-Claude DESTUTT DE TRACY, Quels sont les moyens de fonder la morale chez un peuple?, Paris, H. Agasse, an VI, p. 33-34 : «Personne malheureusement ne peut nier que depuis quelques années, en France, les crimes sont plus nombreux, les passions plus exaspérées, les malheurs particuliers plus multipliés; en un mot, que le désordre de la société est plus grand qu'auparavant. [...] Quelle est la cause de cette triste vérité ? Tous les gens irréfléchis, et c'est le grand nombre, vous répondent que la révolution a démoralisé la nation française : et ils croient avoir rendu raison de tout. Mais qu'entendent-ils par ce mot? Veulent-ils dire que parmi nous la somme du mal moral est augmentée ? Alors [...] ils énoncent simplement le fait, et n'indiquent pas la cause.

Veulent-ils insinuer que le changement de gouvernement a rendu nos mœurs plus dépravées, nos sentiments plus pervers? Alors ils oublient que les sentiments des hommes ne changent point ainsi du jour au lendemain, ni même en un petit nombre d'années. Il est constant, au contraire, que le temps présent est toujours, pour ainsi dire, le disciple du temps antérieur, et que nous sommes mus aujourd'hui par les habitudes, les passions et les idées contractées ou acquises sous l'ancien ordre social. Si telles étaient les causes de nos maux actuels, il faudrait sans hésiter les attribuer tous à cet Ancien Régime si follement regretté. Mais soyons toujours justes. Ce serait outrer les reproches qui lui sont dus, puisque tant qu'il a subsisté, ces habitudes, ces passions et ces idées n'entraînaient pas toutes les mêmes conséquences. "

17. Voir Marc DELEPLACE : «Le discours sur l' «anarchie» en l'an III : entre «Terreur» et "Contre-Révolution»", dans Michel vovelle (dir.), Le tournant de l'an III. Réaction et Terreur blanche dans la France révolutionnaire, Paris, Editions du CTHS, 1997, p. 221-227. L'insurrection populaire est clairement, à cette époque, rejetée du côté de l'anarchie. Mais dans la mesure même où tout bouleversement politique peut $\mathrm{y}$ conduire, certains Républicains sous le Directoire n'hésitent pas non plus à y associer le retour à la monarchie. Voir Ibid., p. 226.

18. Voir Bronislaw BACZKo, Politiques de la Révolution française, p. 325 sq.

19. Ibid., p. 35.

20. Germaine de STAËL, Des circonstances actuelles qui peuvent terminer la révolution et des principes qui doivent fonder la république en France, Paris, Droz, 1979, p. 33 [le titre sera dorénavant abrégé : Des circonstances actuelles] : « On peut attribuer à plusieurs principes les horreurs de la Révolution en France. Les principaux sont les obstacles qu'elle a eus à renverser : les caractères que les abus de l'Ancien Régime avaient formés dans le peuple, le manque absolu de morale publique réduit presque en maxime, enfin la fausse application du principe de la souveraineté du peuple dans le gouvernement représentatif. Aucune de ces causes ne naît de la théorie politique qui compose la doctrine des républicains éclairés. »

21. Benjamin CONSTANT, op.cit.

22. Ibid., De la force du gouvernement actuel, p. 33.

23. Ibid., p. 43-44.

24. Benjamin CONSTANT, Des effets de la Terreur, p. 173.

25. Voir Jean-Luc CHAPPEY, "Révolution, régénération, civilisation: enjeux culturels des dynamiques politiques", dans Jean-Luc CHAPPEY, Bernard GAINOT, Guillaume MAZEAU, Frédéric REGENT, Pierre SERNA, Pour quoi faire la révolution?, Marseille, Agone, 2012, p. 136 : «Régénérer ou civiliser s'inscrit, pour les élites républicaines, dans le programme de dépolitisation des catégories populaires. »

26. Cité par Bronislaw BACZKo, «La constitution de l'an III », dans François AzouVI (dir.), L'institution de la raison, Vrin, EHESS, 1992, p. 26.

27. Voir Benjamin CONSTANT, Des réactions politiques, p. 98. 
28. Germaine de STAËL, Des circonstances actuelles, p. 160.

29. Pierre SERNA, La république des girouettes, Seyssel, Champ Vallon, 2005, p. 435.

30. Jean-Luc CHAPPEY, art.cit., dans Jean-Luc CHAPPEY, Bernard GAINOT, Guillaume MAZEAU, Frédéric REGENT, Pierre SERNA, Pour quoi faire la révolution?, Marseille, Agone, 2012, p. 119.

31. François AZOUVI (dir.), L'institution de la raison, Vrin, EHESS, 1992, p. 15.

32. Antoine-Louis-Claude DESTUTT DE TRACY, Quels sont les moyens de fonder la morale chez un peuple, Paris, H. Agasse, An VI, p. 22.

33. Antoine-Louis-Claude DESTUTT DE TRACY, Commentaire sur l'esprit des lois de Monstesquieu, Paris, Delaunay, 1819 , p. 51.

34. Pierre-Claude-François DAUNOU, "Essai sur l'instruction publique », dans Bronislaw BACZKO (dir.), Une éducation pour la démocratie. Textes et projets de l'époque révolutionnaire, Genève, Droz, 2000, p. 311 : «Pour moi, quand je verrai de telles institutions s'élever et alterner dans nos communes, c'est alors que je croirai en effet à la régénération de la France; je dirai : "la République est fondée, mon pays est libre." "

35. Sur ce point, l'écart entre un Destutt de Tracy et d'autres acteurs de la Révolution, qui avaient conçu la popularisation de la science comme un moyen de régénération est grand. Voir Jean-Luc CHAPPEY, «Enjeux sociaux et politiques de la "vulgarisation scientifique" en Révolution (1780-1810) », Annales historiques de la Révolution française, $\mathrm{n}^{\circ}$ 338, octobre 2004, p. 21.

36. Germaine de STAËL, Des circonstances actuelles, p. 276.

37. Benjamin CONSTANT, De la force du gouvernement actuel, p. 74.

38. Germaine de STAËL, De la littérature, Paris, GF, 1991

39. Germaine de STAËL, op.cit, p. 302 : «L'on s'est persuadé pendant quelque temps, en France, qu'il fallait aussi faire une révolution dans les lettres, et donner aux règles du goût, en tout genre, la plus grande latitude. »

40. Ibid., p. 308.

41. Florence LotTerie, « Madame de Staël et l'esprit de Coppet : une littérature d' "opposition" ? ", dans Jean-Claude BonNET (dir.), L'Empire des Muses. Napoléon, les Arts et les Lettres, Paris, Belin, 2004, p. 139.

42. Jean-Pierre SCHANDELER, «Condorcet et l'invention de la perfectibilité indéfinie. Une contribution aux "sciences morales et politiques" ", dans Bertrand BINOCHE (dir.), L'homme perfectible, Seyssel, Champ Vallon, p. 248.

43. Cette différence entre le niveau individuel et social apparait implicitement lorsque Florence LOTTERIE écrit, dans Progrès et perfectibilité: un dilemme des Lumières françaises (1755-1814), Oxford, Voltaire Foundation, 2006, p. 103 : «La pédagogie est le premier levier de la nouvelle science politique, qui se propose de régénérer la société en rationalisant les conduites et les stratégies individuelles de manière à les conformer aux exigences du régime représentatif. » L'écart entre cette connaissance de comportements rationnels et l'objectif de transformation - voire chez certains, de « régénération » - politique et sociale doit être cependant analysé.

44. Voir Jean-Luc CHAPPEY, « De la science de l'homme aux sciences humaines: enjeux politiques d'une configuration du savoir (1770-1808) », Revue d'histoire des sciences humaines, 2006/2, n 15, p. 51.

45. Voir Jean-Luc CHAPPEY, La société des observateurs de l'homme (1799-1804). Des anthropologues au temps de Bonaparte, Paris, Société des études robespierristes, 2002, p. 57 : chez Cabanis, le médecin philosophe incarne ainsi une nouvelle figure de "spécialiste de la morale». Nous ne nous appesantirons pas ici plus avant sur cette parenté de la politique et de la médecine.

46. Voir Antoine-Louis-Claude DESTUTT DE TRACY, Traité de la volonté humaine et de ses effets, Paris, Fayard, 1994 [1818], p. 409.

47. Antoine-Louis-Claude DESTUTT DE TRACY, Quels sont les moyens de fonder la morale chez un peuple, op.cit., p. 29. 
48. Voir Florence LOTTERIE, Progrès et perfectibilité: un dilemme des Lumières françaises (1755-1814), Oxford, Voltaire Foundation, 2006, p.69: "Le législateur se fait prudent accompagnateur du temps et assure un développement maîtrisé des facultés de l'homme. »

49. Voir Antoine-Louis-Claude DESTUTT DE TRACY, « De la métaphysique de Kant », dans Mémoire sur la faculté de penser. De la métaphysique de Kant et autres textes, Paris, Fayard, 1992, p. 288-289.

50. Laurent CLAUZADE, L'idéologie ou la révolution de l'analyse, Paris, Gallimard, 1998, p. 24.

51. Pierre-Jean-Georges CABANIS, Rapports du physique et du moral de l'homme, t. I, Paris, Crapelet, 1805 , p. 38.

52. Joseph LAKANAL, « Rapport sur l'établissement des écoles normales », dans Bronislaw BACZKO (dir.), op.cit,. p. 474.

53. Ibid., p. 480.

54. Voir Nestor CAPDEVILA, Le concept d'idéologie, Paris, PUF, 2004, p. 37.

55. Germaine de STAËL, De la littérature, Paris, GF, 1991, p. 308.

56. Joseph LAKANAL, Rapport sur l'établissement des écoles normales, dans Bronislaw BACZKO, Une éducation pour la démocratie. Textes et projets de l'époque révolutionnaire, Genève, Droz, 2000, p. 475.

57. Ibidem.

58. Antoine-Louis-Claude DESTUTT DE TRACY, Mémoire sur la faculté de penser, Paris, Fayard, 1992 [1798], p. 69 : « La science qui nous occupe est si neuve, qu'elle n'a point encore de nom. »

59. Voir Antoine-Louis-Claude DESTUTT DE TRACY, Eléments d'idéologie, t. III, Paris, 1818, p. 385 : « Dès qu'on connaît la génération de nos sentiments, on sait les moyens de cultiver les uns et de déraciner les autres. Par conséquent, les principes de l'éducation et de la législation sont à découvert, et la science de l'homme en tant que voulant et agissant est achevée. " Mais cette science « achevée » est précisément encore à l'état de projet et de programme!

60. Joseph LAKANAL, «Rapport sur les écoles normales", Bronislaw BACZKo (dir.), Une éducation pour la démocratie. Textes et projets de l'époque révolutionnaire, Genève, Droz, 2000, p. 479.

61. Voir Antoine-Louis-Claude DESTUTT DE TRACY, Quels sont les moyens de fonder la morale chez un peuple?, p. 20.

62. Jean-Luc CHAPPEY montre qu'une telle inquiétude était présente, chez certains acteurs, dès le début de la Révolution française, notamment chez Lacretelle. Voir Jean-Luc CHAPPEY, «De la science de l'homme aux sciences humaines: enjeux politiques d'une configuration des savoirs (1770-1808), Revue d'Histoire des Sciences Humaines, 2006/2, n 15, p. 51.

\section{RÉSUMÉS}

Le caractère inédit, dans l'histoire de France, d'un régime tel que la République ne suppose pas, chez les Thermidoriens, une régénération instantanée de l'homme par le biais des institutions politiques ou de l'événement révolutionnaire. Si du nouveau doit apparaître en l'homme, celui-ci ne deviendra pas pour autant un " homme nouveau ». Cet article délimite le périmètre de cette nouveauté non révolutionnaire, qui se situe entre la corruption de l'Ancien Régime et la dépravation ou la "vulgarité » occasionnées par la Révolution française. Tout en étant produite par des moyens politiques, la nouveauté spirituelle, morale et intellectuelle ne dépend pas de la seule action du gouvernement. La formation des esprits suppose l'existence de sciences de l'homme, pourtant encore en construction. Cette histoire des sciences n'est pas un reflet de l'histoire politique, mais se conçoit de façon similaire en termes de « rénovation » scientifique 
sur le long terme ou de construction d'une littérature républicaine nouvelle. Ce faisant, cet article montre l'imbrication, mais aussi l'autonomie des nouveautés scientifique, morale et politique.

To the republican authors of the Thermidorian regime, the unique character, in French history, of a political regime such as the republic does not imply the instant regeneration of man thanks to the political institutions. If a transformation occurs in a human being, this does not consenquently mean that the latter will become a "new man". This article sets the boundaries of the perimeter of this non-revolutionary novelty, which lies between the corrupted Ancien Regime and the depravation or vulgarity that result from the French Revolution. Although it is produced by the politic through laws, the public instruction or the republican authors, the moral and intellectual novelty does not depend only on governmental action. The forming of minds implies the prior existence of science of man, even though it is in becoming. This history of science does not reflect the political history but can be conceived similarly in terms of scientific renovation on the long run or the construction of a new republican literature. By doing so, this article shows the imbrications but also the autonomy of scientific, moral and political novelty.

\section{INDEX}

Keywords : Destutt de Tracy, Daunou, Lakanal, Germaine de Staël, Benjamin Constant, depravity, vulgarity, renovation, Revolution, regeneration, perfectibility

Mots-clés : Destutt de Tracy, Daunou, Lakanal, Germaine de Staël, Benjamin Constant, dépravation, vulgarité, rénovation, Révolution, régénération, perfectibilité

\section{AUTEUR}

\section{AYŞE YUVA}

Post-doctorante

Centre Marc Bloch (Berlin)

Centre de Recherches Interdisciplinaires sur les Lumières en Europe (I.Z.E.A.),

Université de Halle

yuva@cmb.hu-berlin.de 